\title{
SUBSCRIPTION INFORMATION
}

\section{$\square$ YES! I want to continue my free subscription to CNS SPECTRUMS}

Name:

Address:

E-mail:

Specialty:

Signature:

Date:

FAX: 212.674.2891

MAIL:

CNS SPECTRUMS

MedWorks Media

333 Hudson Street

7th floor

New York, NY 10013

\section{PAID SUBSCRIPTIONS}

$\square$ PRIMARY PSYCHIATRY

First in Applied Psychiatric Medicine

12 issues per year. One-year subscription rate:

Domestic: \$90; Foreign: \$145; In-training: \$50 (2000)

\section{$\square$ TEN}

\section{The Economics of Neuroscience}

12 issues per year. One-year subscription rate:

Domestic: \$120; Foreign: \$185; In-training: \$75

\section{$\square$ ONE}

\section{Oncology Economics}

12 issues per year. One-year subscription rate:

Domestic: \$120; Foreign: \$185; In-training: \$75

\section{CONTINUINg MEdical EDUCATION}

Category 1 credits for psychiatrists, primary care physicians, and neurologists are available each month in CNS Spectrums, as well as on the MedWorks Media Web site:

\section{www.medworksmedia.com}

To participate, complete and mail the test and registration forms that are included in every issue of CNS Spectrums, or visit our Web site. 


\section{Introduction}

CNS Spectrums is a peer-reviewed journal that publishes original scientific literature and reviews on a wide variety of neuroscientific topics of interest to the clinician. CNS Spectrums publishes 12 issues in 2000 . As the immense prevalence of comorbid diseases among patients seen by psychiatrists and neurologists increases, these physicians will jointly diagnose and treat the neuropsychiatrically ill. Our mission is to provide these physicians with an editorial package that will enhance and increase their understanding of neuropsychiatry; therefore, manuscripts that address crossover issues germane to neurology and psychiatry will be given immediate priority.

\section{Scope of Manuscripts}

CNS Spectrums will consider the following types of articles for publication:

Original Reports: Original reports present methodologically sound original data.

Reviews: Reviews are overview articles that summarize and synthesize the literature on various topics in a scholarly and clinically relevant fashion. Suitable topics include mood disorders, schizophrenia and related disorders, personality disorders, substanceuse disorders, anxiety disorders, neuroscience, psychosocial aspects of psychiatry, child psychiatry, geriatric psychiatry, and other topics of interest to clinicians. nb: Original flowcharts designed to aid the clinician in diagnosis and treatment will be considered for publication in reviews and are encouraged.

Case Reports: Single or multiple case reports will be considered for publication.

Letters to the Editor: Letters will be considered for publication.

\section{Manuscript Submissions}

General information: Four copies of the manuscript should be submitted to Jack M. Gorman, editor (or, in Europe, to Joseph Zohar, international editor), c/o MedWorks Media, 665 Broadway, Suite 805, New York, NY 10012; T: 212.328.0800, F: 212.328.0600. Authors are required to submit their manuscripts on computer disks. If possible, please provide them in MSWord Word for Windows in either a Macintosh or IBM format. (Saving the file in a lower version, eg, MSWord 3.0, is also encouraged.) Disks should be labeled with the word-processing program, title of paper, and first author's name.

Letters of permission to reproduce previously published material: All material reproduced from previously published copyrighted material must be accompanied by a letter of permission from the copyright holder. All such material should include a full credit line (eg, in the figure or table legend) acknowledging the original source. Any citation of unpublished material or personal communication should also be accompanied by a letter of permission for anyone who is not an author of the paper.

Peer review: Authors should provide five names of particularly qualified potential reviewers with no conflict of interest in reviewing the work. Contact information, including complete address, phone, fax numbers, E-mail address, and affiliations, should be included. The corresponding author will be notified by the editors when a decision regarding acceptance has been made. Accepted manuscripts and letters will be edited for clarity and style.

\section{Manuscript Preparation}

Length: Reviews should not exceed 20 manuscript pages $(10,000$ words). Original reports should not exceed 15-25 manuscript pages (6,250 words, maximum). Letters should not exceed 2-6 manuscript pages (1,500 words, maximum). Single case reports should not exceed 10-15 manuscript pages (3,750 words, maximum) and may be submitted with a photograph, if applicable. Diagnostic/treatment algorithms (see Reviews) should contain an extensive introduction, a flowchart or series of graphs that fill 8-12 journal pages, and a concise summary.

Spacing: One space should be left after commas and periods. Manuscripts should also be double-spaced.

Abstract: Authors should provide a brief abstract.

References: American Medical Association style. See the following examples:

1. Jones J. Necrotizing Candida esophagitis. JAMA. 1980;244:2190-2191.

2. Stryer L. Biochemistry. 2nd ed. San Francisco, Calif: WH Freeman Co; 1980:559-596.

Copyright: Materials are accepted for exclusive publication in CNS Spectrums and become the property of CNS Spectrums. Permission to reproduce material must be obtained from the publisher.

\section{Disclosure of Commercial Interests}

The authors must include a statement about all forms of support, including grant and drug company support. Such information may, at the editor's discretion, be shared with reviewers. If the article is accepted for publication, the editors will consult with the authors as to whether this information should be included in the published paper.

Reprints: Authors of reviews and original materials published in CNS Spectrums may order reprints of their articles directly from the publisher, James La Rossa Jr., MedWorks Media, New York, NY 10012; T: 212.328.0800, F: 212.328.0600.

Continuing Medical Education requirements: Authors must submit four multiple-choice questions (two Type A and two Type K) with answers.

\section{Submission Checklist}

1. Original manuscript plus copies

2. Copies of permission letters to reproduce previously published and unpublished material

3. A brief abstract of article.

4. Two multiple-choice questions with answers

5. Disk labeled with the word-processing program, title of paper, and first author's name

6. Names and addresses of five potential reviewers. 


\section{GuIDE to DSM-IV AND ICD-10 CODES}

Dementia of the Alzheimer Type, With Early Onset With Depressed Mood

DSM-IV

ICD-10

Specify if: With Behavioral Disturbance

290.13

F00.03

Specify if: With Behavioral Disturbance

Delirium Due to: Indicate General Medical Condition

Psychotic Disorder Due to: Indicate General Medical Condition With Delusions

With Hallucinations

Mood Disorder Due to: Indicate General Medical Condition

Anxiety Disorder Due to: Indicate General Medical Condition

Amnestic Disorder Due to: Indicate General Medical Condition

Dementia NOS

Amnestic Disorder NOS

Schizophrenia

Schizophrenia-Disorganized Type

Schizophrenia-Catatonic Type

Schizophrenia-Paranoid Type

Schizophrenia-Residual Type

Schizoaffective Disorder

Schizophrenia-Undifferentiated Type

Major Depressive Disorder

Bipolar I Disorder

Bipolar Disorder NOS

Bipolar II Disorder

Mood Disorder NOS

Psychotic Disorder NOS

Autistic Disorder

Asperger's Disorder

Pervasive Developmental Disorder NOS

Anxiety Disorder NOS

Panic Disorder Without Agoraphobia

Generalized Anxiety Disorder

Dissociative Identity Disorder

Dissociative Disorder NOS

Factitious Disorder NOS

Panic Disorder With Agoraphobia

Agoraphobia Without History of Panic Disorder

Social Phobia

Specific Phobia

Obsessive-Compulsive Disorder

Dysthymic Disorder

Depersonalization Disorder

Body Dysmorphic Disorder

Somatization Disorder

Somatoform Disorder NOS

Cyclothymic Disorder

Alcohol Dependence

Cocaine Dependence

Cannabis Dependence

Amphetamine Dependence

Alcohol Abuse

Cannabis Abuse

Cocaine Abuse

Amphetamine Abuse

Stuttering

Anorexia Nervosa

Tic Disorder NOS

Tourette Disorder

Primary Insomnia

Primary Hypersomnia

Sleepwalking Disorder

Dyssomnia NOS

Nightmare Disorder

Parasomnia NOS

Eating Disorder NOS

Bulimia Nervosa

Feeding Disorders of Infancy or Early Childhood

Communication Disorder NOS

Posttraumatic Stress Disorder

Depressive Disorder NOS

Impulse-Control Disorder NOS

Pathological Gambling

Pyromania

Kleptomania

Trichotillomania

Disruptive Behavior Disorder NOS

Attention-Deficit/Hyperactivity Disorder, Combined Type

Attention-Deficit/Hyperactivity Disorder NOS

Learning Disorder NOS

Developmental Coordination Disorder

Narcolepsy

Sleep Disorder Due to: Indicate General Medical Condition

Delirium NOS

290.21

293.0

293.81

293.82

293.83

293.89

294.0

294.8

294.8

295

295.10

295.20

295.30

295.60

295.70

295.90

296

296.80

296.80

296.90

298.9

299.00

299.80

299.80

300.00

300.01

300.02

300.14

300.15

300.19

300.21

300.22

300.23

300.29

300.3

300.4

300.6

300.7

300.81

300.81

301.13

303.90

304.20

304.30

304.40

305.00

305.20

305.60

305.70

307.0

307.1

307.20

307.23

307.42

307.44

307.46

307.47

307.47

307.47

307.50

307.51

307.59

307.9

309.8

311

312.30

312.31

312.33

312.34

312.39

312.9

314.01

314.9

315.9

315.4

347

780

780.09
F00.13

F05.0

F06.2

F06.0

F06

F06.4

FO2.8

FO3

R41.3

F2O

F20.1

F20.2

F20.0

F20.5

F25

F20.3

F32

F30

F39

F31.8

F39

F29

F84

F84.5

F84.9

F41.9

F41

F41.1

F44.81

F44.9

F68.1

F40.01

F40

F40.1

F40.2

F42.8

F34.1

F48.1

F45.2

F45.

F45.9

F34

F10.2

F14.2

F12.2

F15.2

F10.1

F12.1

F14.1

F15.1

F98.5

F50

F95.9

F95.2

F51.0

F51.1

F51.3

F51.9

F51.5

F51.8

F50.9

F50.2

F98.2

F80.9

F43.1

F32.9

F63.9

F63.0

F63.1

F63.2

F63.3

F91.9

F90

F90.9

F81.9

F82

G47.4

G47

F05.9 


\section{FaxBack Response}

Name:

\section{Address:}

\section{E-mail:}

Specialty:

\section{Signature:}

Date:
FAX: 212.674.2891

MAIL:

CNS SPECTRUMS

MedWorks Media

333 Hudson Street

7th floor

New York, NY 10013

Your comments are important to us. This easy-to-use form provides you with the opportunity to express your opinions. Our goal is to make CNS Spectrums your source for practical and clinical neuropsychiatric information. By filling out this FaxBack form, you will enable us to incorporate your views about our editorial content in future issues. Please fill out this form in its entirety. Thank you.

1. On a scale of 1 to 5 ( $1=$ Poor, $5=$ Excellent), please indicate your level of interest and/or satisfaction with the editorial content in this issue.

\section{Cover Story}

$12 \quad 3 \quad 4 \quad 5$

Departments

CNS News

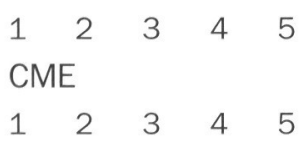

2. Which areas of neuropsychiatry would you like us to cover in the future?
3. Please describe your reading pattern for this issue:

O cover to cover

skim Table of Contents

select items of interest

skim text

did not read

4. On a scale of 1 to 5 ( $1=$ Incomplete, $5=$ Comprehensive), how would you describe the depth of coverage for this issue? $\begin{array}{lllll}1 & 2 & 3 & 4 & 5\end{array}$

5. Any other comments?

6. Please indicate your title:

psychiatrist

neurologist

When you send us this form, you'll receive one complimentary slide library and one complimentary reference material. Please make your selection below.

\section{SLIDE LIBRARY}

Current Uses of Dopamine Agomists

- Momotherapeutic Uses for Dopamine Agomists

Diagnosis and Treatment of Premenstrual Dysphoric Disorder

- Managing Psychiatric Illness in the Elderly

Diagnosis and Treatment of Anxiety Disorders in Children

D Optimal Uses of Antidepressants

New Developments in the Treatment of Epilepsy

Immunogenicity of Botulinum Toxin Therapyy

\section{REFERENCE MATERIALS}

\The Use of Anticomvulsants in the Treatment of Neuropathic Pain

$\square$ Overview of Social Anxiety Disorder (Social Phobia): Recognition and Treatment

- Advances in Diagnosis and Treatment of PTSD

$\square$ Current Treatments of ADHD

$\square$ Current and Emenging Treatments for Cervical Dystomia

The Black Book of Psychotropic Dosing and Monitoring 2000 


\section{EFFFXXRXR R R}

Brief Summary

Indications and Usage: Effexor XR is indicated for the treatment of depression and for the treatment of Generalized Anxiety Disorder ( $G A D$ ). Concomitant use in patients taking monoamine oxidase inhibitors (MAOIs) is contraindicated (see "Warnings"),
Wamings: POTENTLL FOR INTERACTION WITH MONOAMINE OXIDASE INHIBITORS-Adverse reactions, some of WarningS: POTENTLL FOR INTERACTION WITH MONOAMINE OXIDASE INHIBITORS-Adverse reactions, some of
which were serious, have been reported in patients who have recently been discontinued from an MAOI and started on venlafaxine, or who have recentty had venlafaxine therapy discontinued prior to initiation of an
MADI. These reactions have included tremor, myoclonus, diaphoresis, nausea, vomiling, flushing, dizziness, hyperthermia with features resembling neuroleptic malignant syndrome, seazures, and death. In' patients
receiving antidepressants with pharmacological properties simlar to venlafaxine in combination with an receiving antidepressants with pharmacolagical properties similar to venlafaxine in combination with an
MAOl, there have also been reports of serious, sometimes fatal, reactions. For a selective serotonin reuptake
inhibitor, these reactions have included hyperthermia, rigidity myoclonus, autonomic instability with possible inhibitor, these reactions have included hyperthermia, rigidity, myoclonus, autonomic instability with possible
rapid fluctuations of vital signs, and mental status changes that include extreme agitation progressing to delirium and coma. Some cases presented with features resembling neuroleptic malignant syndrome. Severe
hyperthermia and seizures, sometimes fatal, have been reported in association with the combined use of tricyclic antidepressants and MA0ls. These reactions have also been reported in patients who have recently cyclic antidopressants and MAOs. These reactions have aso been reported in patients who of recenty
discontinued these drugs and have been started on an MAOI. The effects of combined use of venlafaxine and
MAOls have not been evaluated in humans or animals. Therefore, because venlafaxine is an inhibitor of both
norepinephrine and sorotonin reuptake, it is recommended that Effexor XR (venlataxine hydrochloride) norepinephrine and serotonin reuptake, it is recommended that Effexor XR (venlataxine hydrochloride)
extended release capsules not be used in combination with an MAOL, or within at least 14 days of discontinu-
ing treatment with an MAOI. Based on the half-life of venlafaxine, at least 7 days should be allowed after ing treatment with an MAOI. Based on the half-life of venlafaxine, at least 7 days should be allowed after
stopping venlafaxine before starting an MAOI-
SUSTANRED HYPERTENSION - Venlafaxine is associated with sustained increases in blood pressure in some patients.

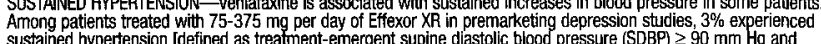
$210 \mathrm{~mm} H \mathrm{Hg}$ above baseline for 3 corisecutive on-therapy visits]. Among patients treated with $75-225 \mathrm{mg}$ per day of
Effexor XR in premarketing GAD studies, $0.4 \%$ (2/476) experienced sustained hypertension. Experience with immediate release venlafaxine showed that sustained hypertension was dose related, increasing from $3-7 \%$ at $100-300 \mathrm{mg}$, $>300 \mathrm{mg} /$ day to fully evaluate the incidence of sustained blood pressure at these higher doses. In premarketing
depression and GAD studies, $0.7 \%$ and $0.4 \%$ of the Effexor $X R$-treated patients, respectively, discontinued treatment depression and $G$ a blood pressure. For patients who

Precautions: GENERAL - Insomnia and Nervousness. Treatment-emergent insomnia and nervousness have been Precautions: GENERAL - Insomnia and Nervousness. Treatment-emergent insomnia and nervousness have been
reported for patients treated with Effexor XR. Insomnia and nevvousness each led to drug discontinuation in $0.9 \%$ of
the patients treated with Effexor XR in Phase 3 depression studies. In Phase 3 GAD trials, insomnia and nervousness led to drug discontinuation in $5 \%$ and $3 \%$, respectively, of the patients treated with Effexor XR.
Changes in AppetiteNeight. Treatment-emergent anorexia has been reported in short-term depression and anxiety Changes in Appetite/Weight Treatment-emergent anorexia has been reported in short-term depression and anxiety
studies. A loss of $5 \%$ or more of body weight occurred in $7 \%$ of Effexor XR-treated and $2 \%$ of placebo-treated
patients in placebo-controlled depression trials. A loss of $7 \%$ or more of body weight occurred in $3 \%$ of the Effexor XRpatients in placebo-controlled depression trials. A loss of $7 \%$ or more of body weight occurred in $3 \%$ of the Effexor XR-
treated and $0 \%$ of the placebo-treated patients in placebo-controlled $G A D$ trials. Activation of Mania/Hypomania. Mania or hypomantia has occurred during short-term depression studies. Effexor XR
should be used cautiously in patients with a history of mania.

Seizures. No seizures occurred among Effexor XR-treated patients in short-term trials. In all premarketing depression
trials with Effexor, seizures were reported in $0.3 \%$ of venlafaxine-treated patients. Use Effexor XR cautiously in patients with a history of seizures. Discontinue in any patient who develops seizures. Suicide: The possibility of a suicide attempt is inherent in depression and may persist until significant remission occurs
Closely supervise high-risk patients during initial duug therapy. Prescriptions for Effexor XR should be written for the smallest quantity of capsules consistent with good patient management to reduce the risk of overdose. The same pre-
cautions observed when treating patients with depression should be observed when treating patients with GAD. cautions observed when treating patients with depression should be observed when treating patients with Gait.
Use in Patients With Concomitant IIness. Premarketing experience with venlafaxine in patients with concomitant sys-
temic illiness is limited. Use Effexor XR cautiously in patients with diseases or conditions that could affect hernodynamic responses or metabolism. Venlafaxine has not been evaluated in patients with recent history of Ml or unstable heart disease. In short-term depression studies electrocardiographic changes in corrected QT interval (QTC) for
Effexor XR-treated patients showed an increase of $4.7 \mathrm{msec}$. In these same trials, the mean change from baseline heart rate for Effexor XR-treated patients was 4 beats per minute. In short-term GAD studies, mean changes in QTC
for Effexor XR-treated patients did not differ significantly from placebo. The mean change from baseline heart rate for for Effexor XR-treated patients did not differ significanty ine and its active metabolites were decreased, thus prolonging the elimination half-lives. A lower dose may be necesSary, uSe with caution in such patients.
INFORMATION FOR PATIENTS - Clinical studies in healthy individuals revealed no clinically significant impairment of psychomotor, cognitive, or complex behavior performance. However, caution patients about operating hazardous
machinery, including automobiles, until they are reasonably sure that venlataxine does not adversely affect their abilimachinery, including automobiles, until they are reasonably sure that ven intend to become pregnant during therapy, or if they are nursing; 2 inform physician about other prescription or over the counter medications they are taking of
plan to take; 3) avoid alcohol while taking Effexor XF; 4) notify their physician if they develop a rash, hives, or related

aABORATORY TESTS: There are no specific laboratory tests recommended.
ORUG INTERACTIONS Cimetidine: Use with caution when administering venlafaxine with cimetidine to patients with pre-existing hypertension or hepatic dystunction, and the elderly. $70 \%$ increase in haloperidol AUC. The haloperidol $\mathrm{C}_{\text {max }}$ increased $88 \%$ when coadministered with venlafaxine, but the haloperidol elimination half-life was unchanged
Drugs Inhibiting Cytochrome P450206 Metabolism. Venlafaxine is metabolized to its active metabolite, 0-desmethylvenlafaxine (ODV, via cytochrome P4502D6. Drugs inhibiting this isoenzyme have the potential to increase plasma
concentrations of ventataxine and decrease concentrations of ODV. However, since the composite plasma levels of venlafaxine and ODV are essentially unchanged in CYP2D6 poor metabolizers, no dosage adjustment is required when venlataxine is coadministered with a CYP2D 0 inhibitor.

The concomitant uss of venlafaxine with a drug treatment(s) that potentially inhibits both CYP2D6 and CYP3A4, the
primary metabolizing enzymes for venlafaxine has not been studied. Therefore caution is advised stould a patient's primary metabolizing enzymes for venlafaxine, has not been studied. Therefore, caution is advised should a patient's
therapy include venlataxine and any agent(s) that produce simultaneous infibition of these two enzyme systems. Dngss Metabolized by Cytochrome P450 soenzymes. Studies indicate that venlafaxine is a relatively weak inhibitor of
CYP2D6. Venlafaxine did not inhibit CYP1A2 and CYP3A4 (in vitro and in vivo), CYP2C9 (in vitro, or CYP2C19 (in vivo) desipramine AUC, $\mathrm{C}_{\max }$ and $\mathrm{C}_{\min }$ increased by about $35 \%$ in the presence of venlafaxine. The $2-\mathrm{OH}$-desipramine AUC increased by 2.5-4.5 Fold. Imipramine did not affect the pharmacokinetics of venlataxine and ODV. The clinical signifiRisperidone- Venlataxine administered under steady-state conditions at $150 \mathrm{mg} /$ day slightly inhibited the CYP2D6mediated metabolism of risperidone (administered as a single $1 \mathrm{mg}$ oral dose) to its active metabolite, 9 -hydroxy-
rispenidone, resulting in an approximate $32 \%$ increase in risperidone AuC. However, venlafaxine coadministration did rispenidone, resulting in an approximate $32 \%$ increase in risperidone AUC. However, venlafaxine coadministration did
not significantty alter the pharmacokinetic profile of the total active moiety (risperidone plus 9 -hydroxyrisperidone). Monoamine Oxidase Inhibitors. See "Contraindications" and "Wamings."
CNS-Active Drugs. Use of venlafaxine with CNS-active drugs has not been systematically evaluated; use caution when Postmarketing Spontaneous Drug interaction Reports: See "ADVERSE REACTIONS," "Postmarketing Reports." 18-month studles in mice given up to $120 \mathrm{mg} / \mathrm{kg} / \mathrm{day}[1.7$ times the maximum recommended human dose (MRHD) $\mathrm{mg} / \mathrm{m}^{2}$ basis] or in $24-$ month studies in rats given up to $120 \mathrm{mg} / \mathrm{kg} /$ day.
Mutagenesis. Venlataxine and $0 \mathrm{OP}$ were not mutagenic in the Ames reverse mutation assay in Salmonella bacteria or the Chinese hamster ovary/HGPRT mammalian cell forward gene mutation assay. Venlataxine was not clastogenic in
several assays. ODV elicted a clastogenic response in the in vivo chromosomal aberration assay in rat bone marrow. Impaiment of Fertifity. No effects on reproduction or fertility in rats were noted at oral doses of up to 2 times the PREGNANCY - Teratogenic Effects - Pregnancy Category $c$. Reproduction studies in rats given 2.5 times, and rabbits given 4 times the MRHD ( $m g / \mathrm{m}^{2}$ basis) revealed no mallormations in offspring. However, in rats given 2.5 times the first 5 days of lactation when dosing began during pregnancy and continued until weaning. There are no adequate and well-controlled studies in pregnant women; use Effexor XR during pregnancy only if clearly needed.
LABOR, DELIVERY, NURSING - The effect on labor and delivery in humans is unknown. Venlafaxine and ODV have been reported to be excreted in human milk. Because of the potential for serious adverse reactions in nursing infants
from Effexor $\mathrm{XR}$, a decision should be made whether to discontinue nursing or to discontinue the drug, taking into account the importance of the drug to the mother

GERIATRIC USE- Approximately $4 \%$ and $3 \%$ of Effexor XR-treated patients in placebo-controlled premarketing Gepression and GAD trials, respectively, were 65 years of age or over. Of 2899 ? Effexor-treated patients in premarketing phase depression studies, $12 \%$ were 65 years of age or over. No overall differences in effectiveness or satety cannot be ruled out. As with other antidepressants, several cases of hyponatremia and syndrome of inappropriate
antidiuretic hormone secretion (SIADH) have been reported, uSually in the elderly.
Adverse Reactions: ASSOCIATED WTH DISCONTINNLATION OF TREATMENT - Approximately $11 \%$ and $23 \%$ of Effexor XR Adverse Reactions: ASSOCIATED WTH DISCONTINUATION OF TREATMENT-APproximately $11 \%$ and $23 \%$ of Effexor
patients in placebo-controlled clinical depression and GAD trials, respectively, discontinued treatment due to an
adverse event. The most common events leading to discontinuation in at least $1 \%$ of patients and at least twice that of placebo in depression trials included: nausea, anorexia, dry mouth, dizziness, insomnia, and somnolence, in U.S. placebo-controlled depression trials included: hypertension, diarmea, paresthesia, tremor, abnormal (mostly blurred)
vision, and abnormal (mostly delayed) ejaculation; in GAD trials included: headache, asthenia, vasodilation, nausea

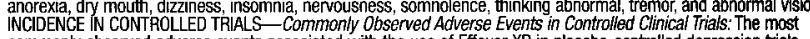
commonly observed adverse events associated with the use of Effexor XR in placebo-controlled depression tials,
(incidence of $5 \%$ or greater and incidence for Effexor XR at least twice that for placebo): nausea (31\% vs. $12 \%$, dizziness $(20 \%$ vs. $9 \%$, somnolence $(17 \%$ vs. $8 \%)$, abnormal ejaculation $(16 \%$ vs. $<1 \%)$, sweating $(14 \%$ vs. $3 \%$, dry
mouth (12\% vs. $6 \%)$, nervousness $(10 \%$ vs. $5 \%$, anorexia $(8 \%$ vs. $4 \%)$, abnormal dreams $(7 \%$ vs. $2 \%)$, and treernor (5\% vS. $2 \%$ ). In U.S. placebo-controlled depression trials, the following were also reported with an incidence of at
least $5 \%$ and at least twice that for placebo: impotence, anorgasmia, decreased libido, constipation, flatulence, insomnia, nervousness, tremor, abnormal vision, hypertension, vasodilation, and yawning. The most commonly observed incidence for Effexor XR at least twice that for placebo): nausea ( $43 \%$ vs. $11 \%)$, dry mouth $(23 \%$ vs. $5 \%$ ), insomnia

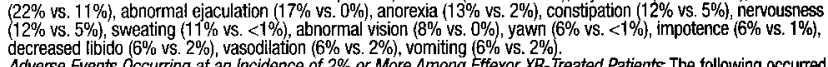
Adverse Events 0 ccurring at an Incidence of $2 \%$ or More Among Effexor XR-Freated Patients. The following occurred in short-term, placebo-controlled depression trials (up to 12 weeks) with doses of 75 to $225 \mathrm{mg} / \mathrm{day}$, at a trequency of $2 \%$ or more and greater than placebo. Body as a Whole: asthenia. Cardiowascular: vasodilation, hypertension. dizziness, somnolence, insomnia, dry mouth, nervousness, abnormal dreams, temor, depression, paresthesia,
decreased libido, agitation. Respiratory System: pharyngitis, yawn. Skin: sweating. Special Senses: abnormal vision. Urogenital System: abnormal ejaculation, impotence, anorgasmia (female). The following occurred in shortand, greater than placebo. Body as a Whole: asthenia infection, abdominal pain, fever, neck pain, chills. Cardiovascular: vasodilation, tactyycardia. Digestive: nausea, anorexia, diarnea, constipation, vomiting, flatulence,
Musculoskeletal System: myalgia. Nervous System: dry mouth, insomnia, dizziness, somnolence, nervousness, Musculoskeletal System: myaigia. Nervous System: dry mouth, insomnia, dizziness, somnolence, nervousness,
decreased libid, abnormal dreams, tremor, paresthesia, thinking abnommal, trismus twitching . Respiratory System
rhinitis, yawn, cough increased. Skin: sweating. Special Senses: abnormal vision. Uragenitail System: abnormal

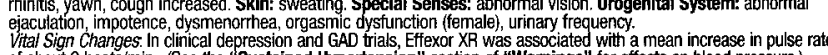
of about 2 beats/min. (See the "Sustained Hypertension" section of "Wamings" for effects on blood pressure.) of about 2 beats/min. (See the sustained Hypertension" section of "Wamings" for eftects on blood pressure.) ECG Changes. (See the "Use in Patients With Concomitant illnesses" section of "Precautions")

OTHER EVENTS OBSERVED DURING THE PREMARKETING EVALUATION OF EFFEXOR ANDD EFFEXOR XR-During premarketing assessment, multiple doses of Effexor XR or Effexor were administered to 4174 patients, and the following
adverse events were reported. Note: "frequent" $=$ events occurring in at least $1 / 100$ patients; "infrequent" $=1 / 100$ to $1 / 1000$ patients; "rare" = fewer than $1 / 1000$ patients. It is important to emphasize that although the events occurred during treatment with venlataxine, they were not necessarily caused by it.
Body as a whole - Frequent: chest pain substemal; Infrequent: face edema, intentional injury, malaise, moniliasis, Bock rigidity, pelvic pain, photosensitivity reaction, suicide attempt; Rare: appendicitis, carcinoma, cellulitis, withdrawal syndrome. Cardiovascular system - Frequent: migraine, postural hypotension; Infrequent: angina pectoris, arthybophlebitis; Rare: arteritis, first-degree atrioventricular block, bigeminy, bradycardia, bundle branch block, cerebral ischemia, coronary artery disease, congestive heart failure, heart arrest, mitral valve disorder, mucocutaneous hemor-
ihage, myocardial infarct, pallor. Digestive system - Frequent: eructation, increased appetite; Infrequent: bruxism, inage, myocardial infarct, pallor. Digestive system - Frequent: eructation, increased appetite; Infrequent: bruxism,
colitis, dysphagia, tongue edema, esophagits, gastritis, gastroenteritis, gastrointestinal ulcer, gingivitis, glossitis, rectal colits, dysphagia, tongue edema, esophagits, gastritis, gastroenteritis, gastrointestinal ulcer, gingivits, glosition,
hemorrhage, hemornhoids, melena, stomatitis, mouth ulceration; Rare: cheilitis, cholecystitis, choleithiasis, hematemesis, gastrointestinal hemorrhage, gum hemorrhage, hepatitis, ileitis, jaundice, intestinal obstruction, oral moniliasis, proctitis, increased salivation, soft stools, tongue discoloration. Endocrine system - Rare: goiter, hyperthy-
roidism, hypothyroidism, thyroid nodule, thyroiditis. Hemic and lymphatic system - Frequent: ecchymosis; Infrequent anemia, leukocytosis, leukopenia, lymph hadenopathy, thrombocythemia, thrombocytopenia; Rare: basophilia, cyanosis,
essinophilia, lymphocytosis. Metabolic and nutritional - Frequent edema, weight gain; Infrequent: alkaline phosphatase increased, glycosuria, hyperchoiesteremia, hyperglycemia, hyperuricemia, hypoglycemia, hypokalemia, SGOT dehydration, gout hemochronatosis, hypercalcinuria, hyperkalemia, hyperlipemia, hyperphosphatemia, hyponatremia, dehydration, gout, hemochromatosis, hypercalcinuria, hyperkalemia, hyperlipemia, hyperphosphatemia, hyponatremia,
hypophosphatema, hypoproteinemia, SGPT increased, uremnia. Musculoskeletal system - Frequent arthralgia; ical fracture, myopathy, osteoporosis, osteosclerosis, mheumatoid arthritis, tendon rupture. Nervous system - Frequent amnesia, confusion, depersonalization, emotional lability, hypesthesia, vertigo; Infrequent: apathy, ataxia, circumoral
paresthesia, CNS stimulation, euphoria, hallucinations, hostility, hyperesthesia, hyperkinesia, hypotonia, incoordination, iibido increased, manic reaction, myoclonus, neuralgia, neuropatthy, paranoid reaction, psychosis, seizure, abnormal speech, stupor, Rare: akathisia, akinesia, alcohol abuse, aphasia, bradykinesia, buccoglossal syndrome, cerebrovas-
cular accident, loss of consciousness, delusions, dementia, dystonia, facial paralysis, abnormal gaít, Guillain-Barré Syndrome, hypokinesia, neuritls, nystagmus, psychotic depression, reflexes decreased, reflexes increased, suicidal
ideation, torticollis. Respiratory system - Frequent: dyspnea; lnfrequent: asthma, chest congestion, epistaxis, hyperventilation, laryngismus, laryngitis, pneumonia, voice alteration; Rare: atelectasis, hemoptysis, hypoventilation, hypoxia pleurisy, pulmonary enmbolus, sleep apnea. Skin and appendages - Frequent, rash, pryuritus; Infrequent: acne,
alopecia brittle nails, contact dermattis, dry skin, eczema, skin hypertrophy, maculopapular rash, psonasis, urticari Rare: erythremia nodosum, exfoliative dermatitis, lichenoid dermatitis, hair, discoloration, skin discoloration, furunculosis, hirsutism, leukoderma, pustular rash, vesiculobullous tash, seborthea, skin atrophy, skin strlae. Special senses Frequent: abnormality of accommodation, mydriasis, taste perversion; Infrequent cataract, conjunctivitis, comeal
lesion, diplopia, dry eyes, exophthalmos, eye pain, hyperacusis, otitis media, parosmia, photophobia, taste loss, visual junctival hemorrhage, keratitis, labyrinthitis, miosis, papilledema, decreased pupillary reflex, ottitis externa, scleritis, uveitis. Urogenital system - Frequent: metrormagia, ${ }^{*}$ prostatitis, ${ }^{*}$ urination impaired, vaginitis*; Infrequent albumin-

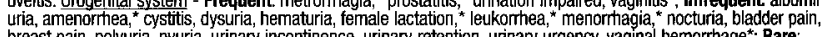
breast pain, polyuria, pyuria, urinary incontinence, urinary retention, urinary urgency, vaginal hemorrhage ${ }^{*} ;$ Rare:

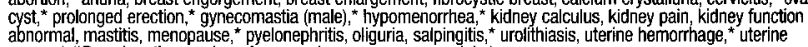
Spasm. (*Based on the number of men and women as appropriate)
Postmarketing Reports: Voluntary reports of other adverse events temporally associated with the use of Effexor (the immediate release form of venlafaxine) that have been received since market introduction and that may have no nia, congenital anomalies, CPK increased, deep vein thrombophlebitis, delirium, EKG abnomalitites (such as atrial fibrillation, supraventricular tachycardla, ventricular extrasystoles, ventricular tachycardia), epidermal necrosis/StevensJohnson Syndrome, enythema multiforme, extrapyramidal symptoms (including tardive dyskinesia), hemorrthage
(including eye and gastrointestinal bleeding), hepatic events (including GGT elevation; abnormalities of unspectried liver function tests; liver damage, necrosis, or tailure; and fatty liver), involuitary movements, $\mathrm{LH}$ increased, neu-
roleptic malignant syndrome-like events (including a case of a 10 -year-old who may have been taking cos dose), and syndrome of inappropriate antidiuretic hormone secretion (usually in the elderly). seizures, following the addition of venlafaxine. There have been reports of increases in prothrombin time, partial thromboplastin time, or INR when venlafaxine was given to patients receiving warfarin therapy. drug abuse and observe such patients closely for signs of venlataxine misuse or abuse (e.g., development of tolerance, incrementation of dose, drug-seeking behavior) $(6 \mathrm{~g}$ of Effexor XR with $2.5 \mathrm{mg}$ of lorazepam, and $2.85 \mathrm{~g}$ of Effexor XP). Both recovered without sequelae. In premarketing evaluation of Effexor, there were 14 reports of acute overdosage (highest dose was $6.75 \mathrm{~g}$ ). All patients
recovered without sequelae. Most patients reported no symptoms. Symptoms observed included somnolence, generalized convulsions, prolongation of $0 T \mathrm{TC}$ to $500 \mathrm{msec}$ ( (compared with $405 \mathrm{msec}$ at baseline) in one case, and \begin{tabular}{l}
$(0.75 \mathrm{~g}$ of Effexor $X R$ and $200 \mathrm{mg}$ of paroxetine and $50 \mathrm{mg}$ of zolpidem, and $1.2 \mathrm{~g}$ of Effexor XR). Both recovered \\
\hline
\end{tabular} without sequelae.
in postmarketing experience, there have been reports of fatalities in patients taking overdoses of venlafaxine, predominantly in combination with alcohol and/or other drugs.
Treatment should consist of those general measures employed in the management of overdosage with any Treatment should consist of those genera measures employed in the management of overdosage with any
antidepressant. Ensure an adequate airway, oxygenation and ventiation. Monitor cardiac rithy and vital signs. Genepral supportive and symptomatic measures are also recommended. Induction of emesis is not recommended. Gastric lavage with a large bore orogastric tube with appropriate airway protection, if needed, may be indicated if
performed soon after ingestion or in symptomatic patients. Activated charcoal should be administered. Due to the large volume of distribution of this drug, forced diuresis, dialysis, hemoperfusion, and exchange transfusion are unlikely to be of benefit. No specific antidotes for venlataxine are known. In managing overcosage, consider the
possibility of multiple drug involvement. The physician should consider contacting a poison control center for additional information on the treatment of any overdose. Telephone numbers for certified poison control centers
are listed in the Physicians' Desk Reference (PDR). are listed in the Physicians' Desk Reference (PDR). discontinuation of an MAOI and initiation of therapy with Effexor XR. In addition, at least 7 days should be allowed after stopping Effexor XR before starting an MAO/ (See "Contraindications"
Please consult full prescribing information for detailed dosing instructions.
This brief summary is based on the circular 4876-4, issued March 22, 1999.

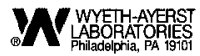

(c) 2000, Wyeth-Ayerst Laboratories 

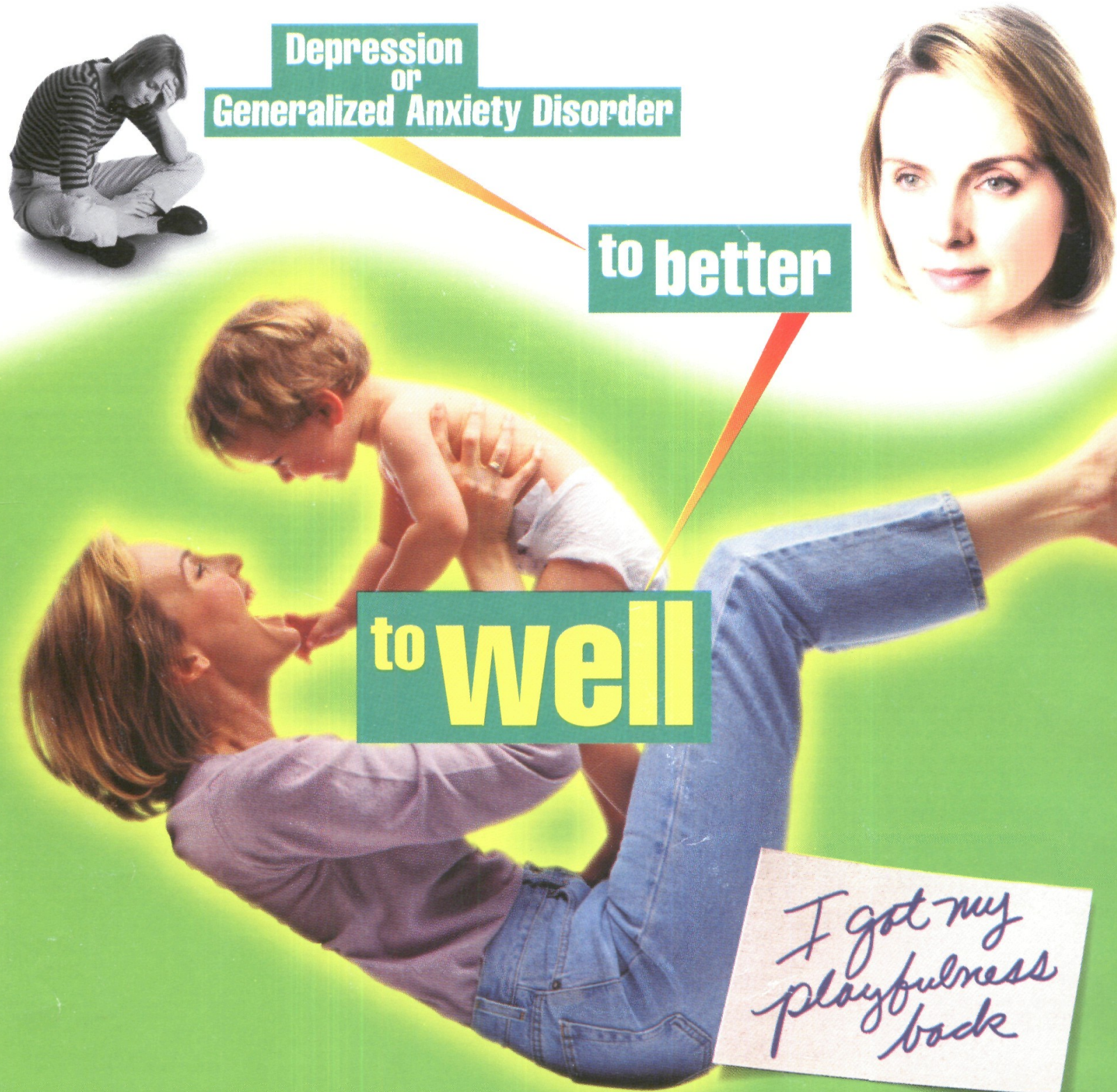

\section{Fet your patients bayond better}

- Working on both serotonin and norepinephrine, the unique formulation of EFFEXOR XR offers more of your patients the ability to achieve remission-full symptom resolution. ${ }^{1,2}$

\section{Need proot? Gall 1-888-EFFEXOR XR.}

\section{Visit us at www.EFFEXORXR.com}

Please see brief summary of Prescribing Information on the next page.

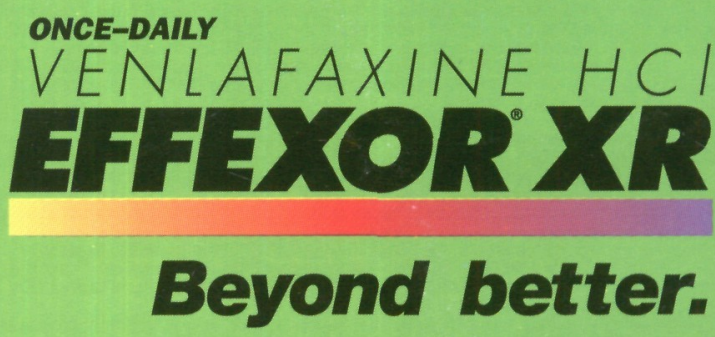

The efficacy and safety of EFFEXOR XR for pediatric use have not been established.

EFFEXOR XR is contraindicated in patients taking monoamine oxidase inhibitors (MAOIs). EFFEXOR XR should not be used in combination with an MAOI or within at least 14 days of discontinuing treatment with an MAOI; at least 7 days should be allowed after stopping EFFEXOR XR before starting an MAOI.

The most common adverse events reported in EFFEXOR XR placebo-controlled depression trials (incidence $\geq 10 \%$ and $\geq 2 \times$ that of placebo) were nausea, dizziness, somnolence, abnormal ejaculation, sweating, dry mouth, and nervousness; and in GAD trials were nausea, dry mouth, insomnia, abnormal ejaculation, anorexia, constipation, nervousness, and sweating. Treatment with venlafaxine is associated with sustained increases in blood pressure (BP) in some patients. Three percent of EFFEXOR XR patients in depression studies (doses of 75 to $375 \mathrm{mg} /$ day) and $0.4 \%$ in GAD studies (doses of 75 to $225 \mathrm{mg} /$ day) had sustained $\mathrm{BP}$ elevations. Less than 1\% discontinued treatment because of elevated BP. Regular BP monitoring is recommended. 